1 Hacettepe Journal of Mathematics and Statistics

$\bigcap$ Volume 46 (6) (2017), 1093-1104

\title{
A structure theorem of left regular cyber-groups
}

\author{
Ying Yuan Xueming Ren* and K. P. Shum ${ }^{\dagger}$
}

\begin{abstract}
An abundant semigroup $S$ is a superabundant semigroup if each $\mathcal{H}^{*}$ class of $S$ contains an idempotent. We call a superabundant semigroup a left regular cyber-group if the set of its idempotents forms a left regular band. After the investigation of the properties of superabundant semigroups, we establish a structure theorem for the left regular cybergroups by using the newly defined left twist product of semigroups.
\end{abstract}

Keywords: Completely regular semigroups; Superabundant semigroups; Left regular cyber-groups.

2000 AMS Classification: 20M10

Received: 13.12.2016 Accepted : 12.01.2017 Doi : 10.15672/HJMS.2017.425

\section{Introduction}

Recall that the generalized Green ${ }^{*}$ relation $\mathcal{L}^{*}$ on a semigroup $S$ is defined by $a \mathcal{L}^{*} b$ if and only if the elements $a, b$ of $S$ are $\mathcal{L}$-related in some oversemigroup of $S$. The generalized Green ${ }^{*}$ relation $\mathcal{R}^{*}$ is dually defined, and also the generalized Green* relation $\mathcal{H}^{*}=\mathcal{L}^{*} \wedge \mathcal{R}^{*}$. According to J. B. Fountain [4], we call a semigroup $S$ abundant if every $\mathcal{L}^{*}$-class and every $\mathcal{R}^{*}$-class of $S$ contains an idempotent. Clearly, all regular semigroups are abundant and in this case, we see that $\mathcal{L}^{*}=\mathcal{L}$ and $\mathcal{R}^{*}=\mathcal{R}$, where $\mathcal{L}$ and $\mathcal{R}$ are the usual Green relations on a semigroup $S$. In [4], J. B. Fountain first introduced and studied a superabundant semigroup, which is a semigroup $S$ in which every $\mathcal{H}^{*}$-class of $S$ contains an idempotent. It is easy to see that abundant semigroups and superabundant semigroups are natural generalizations of regular semigroups and completely regular semigroups, respectively.

In the theory of regular semigroups the class of completely regular semigroups and some of its subclasses play a crucial role from the richness of their structures (see Petrich

\footnotetext{
*Department of Mathematics, Xi'an University of Architecture and Technology, Xi'an 710055, China

Email : xmren@xauat.edu.cn

${ }^{\dagger}$ Institute of Mathematics, Yunnan University, Kunming 650091, China

Email : kpshum@ynu.edu.cn

The research is supported by National Natural Science Foundation of China Grant (No:11471255)
} 
and Reilly [9]). In view of the theory of superabundant semigroups, it becomes very natural to ask to what extent the structure of superabundant semigroups and its special subclass can be determined by the properties of its idempotents. In particular, we call a superabundant semigroup whose set of idempotents forms a subsemigroup a cyber-group. A left cyber-group in the class of abundant semigroups was first studied by X. J. Guo and K. P. Shum in 2004. A structure theorem of cyber-groups was also established by Ren and Shum [13].

In this paper, we consider a superabundant semigroup in which the set of all idempotents becomes a left regular band, namely the left regular cyber-group. Thus, a left regular cyber-group is an analogue for abundant semigroups of left regular orthogroups in the class of completely regular semigroups. After proving some characterization theorems of cyber-groups, we establish a structure theorem of left regular cyber-groups by using the newly defined left twist product of semigroups. As an application of the above structure theorem of left regular cyber- groups, we construct a non-trivial example of left regular cyber-groups.

The readers are referred to the known monographs of J. M. Howie, see ([6],[7]), M. Petrich and N. Reilly [9] for the notations and terminologies not given in this paper.

\section{Preliminaries}

We begin with some basic results, which will be frequently used in this paper. Let $S$ be a semigroup. Firstly, we give an alternative description of $\mathcal{L}^{*}$ from [7] and [4].

2.1. Lemma. Let $S$ be a semigroup and $a, b \in S$. Then the following conditions are equivalent:

(i) $a \mathcal{L}^{*} b$;

(ii) for all $x, y \in S^{1}, a x=a y$ if and only if $b x=b y$.

As a direct consequence of Lemma 2.1, we immediately obtain the following corollary.

2.2. Corollary. Let $S$ be a semigroup, $a \in S$, and $e$ be an idempotent of $S$. Then the following conditions are equivalent:

(i) $a \mathcal{L}^{*} e$;

(ii) $a e=a$ and for all $x, y \in S^{1}, a x=a y$ implies $e x=e y$.

It is clear that on any semigroup $S$ we always have $\mathcal{L} \subseteq \mathcal{L}^{*}$ and also for the regular elements $a, b$ of $S$, we have $a \mathcal{L}^{*} b$ if and only if $a \mathcal{L} b$. In particular, if $S$ is a regular semigroup, then we have $\mathcal{L}^{*}=\mathcal{L}$.

The dual results for $\mathcal{R}^{*}$ also hold in a semigroup $S$. We use $\mathcal{H}^{*}$ to denote the intersection of the relations $\mathcal{L}^{*}$ and $\mathcal{R}^{*}$. The $\mathcal{L}^{*}$-class containing the element $a$ of a semigroup $S$ is always denoted by $L_{a}^{*}$ or $L_{a}^{*}(S)$. In case of ambiguity. The corresponding notation is adopted for the classes of the other relations.

Assume that $S$ is an abundant semigroup and $a$ is an element of $S$. Then we denote an arbitrary idempotent of $L_{a}^{*}$ and $R_{a}^{*}$ by $a^{*}$ and $a^{+}$, respectively. In particular, if $S$ is a superabundant semigroup, then it is clear that every $\mathcal{H}^{*}$-class of $S$ is a cancellative monoid and so, for any $a \in S$, the identity of $H_{a}^{*}$ which is the $\mathcal{H}^{*}$-class contains the element $a$ is denoted by $a^{0}$.

We call a semigroup $S$ a semilattice $Y$ of semigroups $S_{\alpha}(\alpha \in Y)$ if there exists an epimorphism $\varphi$ of $S$ onto the semilattice $Y$ with $\alpha \varphi^{-1}=S_{\alpha}(\alpha \in Y)$. In this case, we usually write $S=\left(Y ; S_{\alpha}\right)$. 
Let $T$ be a cancellative monoid. Let $I$ and $\Lambda$ be a left zero band and a right zero band, respectively. Then we call the direct product $I \times T \times \Lambda$ of $T, I$ and $\Lambda$ a rectangular monoid; The direct products $I \times T$ and $T \times \Lambda$ are also called a left rectangular monoid and a right rectangular monoid, respectively.

In view of the results given by Ren and Shum in [13], we give below a description for the cyber-groups in the following lemma.

2.3. Lemma. ${ }^{[13]}$ A semigroup $S$ is a cyber-group if and only if $S$ is a semilattice $Y$ of rectangular monoids $S_{\alpha}=I_{\alpha} \times T_{\alpha} \times \Lambda_{\alpha} \quad(\alpha \in Y)$ such that for any $\alpha \in Y$ and $a=(i, x, \lambda) \in S_{\alpha}$, we have $a \mathcal{H}^{*}(S) a^{0}$, where $a^{0}=\left(i, e_{\alpha}, \lambda\right)$ and $e_{\alpha}$ is the identity of a cancellative monoid $T_{\alpha}$.

At the end of this section, we recall an interesting subclass of cyber-groups (superabundant semigroups), namely, the $C$ - $a$ semigroups. The following statements on $C-a$ semigroups is directly taken from [3] and [13].

2.4. Lemma. Let $S$ be an adequate semigroup with semilattice of idempotents $E(S)$. Then the following conditions are equivalent:

(i) each $\mathcal{H}^{*}$-class of $S$ contains an idempotent;

(ii) $E$ is central in $S$;

(iii) for all elements $a$ of $S, a^{*}=a^{+}$;

(iv) $\mathcal{L}^{*}=\mathcal{R}^{*}=\mathcal{H}^{*}$;

(v) $S$ is a strong semilattice of cancellative monoids.

An adequate semigroup satisfying one of the above conditions is said to be a $C$ - $a$ semigroup. It is easy to see that a semigroup $S$ is a $C$ - $a$ semigroup if and only if $S$ is superabundant and $E$ is central in $S$. Clearly, a $C$-a semigroup is a natural generalization for abundant semigroups of a Clifford semigroup.

\section{Definitions and characterization theorems}

In this section, we concentrate on some special subclases of superabundant semigroups. We first recall the following notation for some of varieties of bands.

$\begin{array}{lll}\mathcal{S} L & \text { semilattices } & x y=y x \\ \mathcal{L} R B & \text { left regular bands } & x y=x y x \\ \mathcal{R} L B & \text { right regular bans } & y x=x y x\end{array}$

We formulate the following basic definition.

3.1. Definition. A superabundant semigroup $S$ is called a left (right) regular cybergroup if the set of its idempotents forms a left (right) regular band.

It is easy to see that a left (right) regular orthogroup in the class of completely regular semigroups is a left( right) regular cyber-group and so a left (right) regular cyber-group is a natural generalization of a left (right) regular orthogroup in the class of regular semigroups for abundant semigroups. A characterization theorem of a left regular cybergroup is given below:

3.1. Theorem. A semigroup $S$ is a left regular cyber-group if and only if $S$ satisfies the following conditions:

(i) $S$ is a semilattice $Y$ of left rectangular monoids $S_{\alpha}=I_{\alpha} \times T_{\alpha}(\alpha \in Y)$;

(ii) For all $\alpha \in Y$ and $a=(i, x) \in S_{\alpha}, a \mathcal{H}^{*}(S) a^{0}$, where $a^{0}=\left(i, e_{\alpha}\right)$ and $e_{\alpha}$ is the identity of the cancellative monoid $T_{\alpha}$; 
(iii) For any $a^{0}, b^{0} \in E(S), a^{0} b^{0}=a^{0} b^{0} a^{0}$.

Proof. By using Lemma 2.3 and noting the fact that if $E(S)$ is a left regular band then every $S_{\alpha}$ given in Lemma 2.3 becomes a left rectangular monoid, that is, $S_{\alpha}=$ $I_{\alpha} \times T_{\alpha}(\alpha \in Y)$, the proof follows directly by using a similar argument as described in Lemma 2.3 (in detail, see [13]).

Following the notation in Section 2, and using Lemma 2.3 and Theorem 4.3 in [13], we now express a superabundant semigroup or a cyber-group $S$ by $S=\left(Y ; S_{\alpha}\right)$.

A fundamental property of cyber-groups can be easily observed in the following lemma.

3.2. Lemma. Let $S=\left(Y ; S_{\alpha}\right)$ be a cyber-group. Let $\alpha, \beta \in Y$ be such that $\alpha \leqslant \beta$. Then $E\left(S_{\alpha}\right)$ is a rectangular band and for any $a, b \in S_{\alpha}, e \in E\left(S_{\beta}\right)$, we have $a e b=a b$.

Proof. It is evident from Lemma 2.3 that $E\left(S_{\alpha}\right)$ is a rectangular band for every $\alpha \in Y$. Suppose that $a, b \in S_{\alpha}$ and $e \in E\left(S_{\beta}\right)$. Again by Lemma 2.3, it is clear that $a^{0} e \in E\left(S_{\alpha}\right)$. Because $E\left(S_{\alpha}\right)$ is a rectangular band, we immediately have $a b=a\left(a^{0} b^{0}\right) b=a\left(a^{0} \cdot a^{0} e\right.$. $\left.b^{0}\right) b=a\left(a^{0} e b^{0}\right) b=a e b$.

\section{Structure of left regular cyber-groups}

In this section, we establish a structure theorem of left regular cyber-groups.

We use the notation $\lambda_{a}$ to denote the inner left translation on a semigroup $S$ determined by $a \in S$ and we use the symbol End $(S)$ to denote the semigroup of endomorphisms of a semigroup $S$ operating on left, respectively.

Let $Y$ be a semilattice. Let $T=\left[Y ; T_{\alpha} ; \theta_{\alpha, \beta}\right]$ be a strongly semilattice of cancellative monoids $T_{\alpha}(\alpha \in Y)$, that is, $T$ is a $C$ - $a$ semigroup. Now, let $I=\bigcup_{\alpha \in Y} I_{\alpha}$ be a semilattice decomposition of a left regular band so that $I$ is decomposed into some left zero bands $I_{\alpha}$, for each $\alpha \in Y$. Now we form the direct product $S_{\alpha}=I_{\alpha} \times T_{\alpha}$ and assume that $1_{\alpha} \in I_{\alpha}$. Define

$$
\sigma: T \longrightarrow \operatorname{End}(I)
$$

by $t \mapsto \sigma_{t}$ to be the mapping satisfying the following conditions:

$\left(C_{1}\right)$ For all $t \in T_{\alpha}$ and $\beta \in Y, \sigma_{t}\left(I_{\beta}\right) \subseteq I_{\alpha \beta}$;

$\left(C_{2}\right) \sigma_{e_{\alpha}}=\lambda_{1_{\alpha}}$, where $\left(1_{\alpha}, e_{\alpha}\right)$ is a given element of $S_{\alpha}$ for each $\alpha \in Y$;

$\left(C_{3}\right)$ For all $s \in T_{\alpha}, t \in T_{\beta}, i \in I_{\alpha \beta}, \lambda_{i} \sigma_{s} \sigma_{t}=\lambda_{i} \sigma_{s t}$;

$\left(C_{4}\right)$ For all $(i, a) \in S_{\alpha},(j, s) \in S_{\beta}^{1}$, and $(k, t) \in S_{\gamma}^{1}$, if $i \sigma_{a}(j)=i \sigma_{a}(k)$ and $a s=a t$, then we have $i \sigma_{e_{\alpha}}(j)=i \sigma_{e_{\alpha}}(k)$ and $e_{\alpha} s=e_{\alpha} t$, where $e_{\alpha}$ is the identity element of $T_{\alpha}$.

On the set $S=\bigcup_{\alpha \in Y} S_{\alpha}$, we define a multiplication "॰" by the following equation.

$$
(i, s) \circ(j, t)=\left(i j^{\sigma_{s}}, s t\right)
$$

for any $(i, s),(j, t) \in S$, where $j^{\sigma_{s}}=\sigma_{s}(j)$. To see that the multiplication "o" given by the formula (4.1) above is well-defined, we assume that $(i, s) \in S_{\alpha},(j, t) \in S_{\beta}$ for some $\alpha, \beta \in Y$. Then, by the condition $\left(C_{1}\right)$, it is clear that $j^{\sigma_{s}} \in I_{\alpha \beta}$ and so $i j^{\sigma_{s}} \in I_{\alpha \beta}$. Since $T$ is a strong semilattice of cancellative monoids $T_{\alpha}(\alpha \in Y)$, it is evident that $s t \in T_{\alpha \beta}$. Thus, the product $\left(i j^{\sigma_{s}}, s t\right)$ belongs to $S_{\alpha \beta} \subseteq S$.

We first state the following lemma.

4.1. Lemma. The above binary operation "○" defined on the set $S=\bigcup_{\alpha \in Y} S_{\alpha}$ is associative. 
Proof. Suppose that $(i, s) \in S_{\alpha},(j, t) \in S_{\beta}$ and $(k, g) \in S_{\gamma}$ for some $\alpha, \beta, \gamma \in Y$. Then, it is clear from the condition $\left(C_{1}\right)$ that $j^{\sigma_{s}} \in I_{\alpha \beta}$ and so $i j^{\sigma_{s}} \in I_{\alpha \beta}$. Now, by the definition of "०", we immediately deduce the following equality:

$$
\begin{array}{rlrl}
{[(i, s) \circ(j, t)] \circ(k, g)} & =\left(i j^{\sigma_{s}}, s t\right) \circ(k, g) & \\
& =\left(i j^{\sigma_{s}} k^{\sigma_{s t}},(s t) g\right) & & \\
& =\left(i j^{\sigma_{s}} \cdot l \cdot k^{\sigma_{s t}}, s t g\right) & & \text { (some } \left.l \in I_{\alpha \beta}\right) \\
& =\left(i j^{\sigma_{s}} \cdot \lambda_{l} k^{\sigma_{s t}}, s t g\right) & & \text { (since } \left.l \in I_{\alpha \beta}\right) \\
& =\left(i j^{\sigma_{s}} \cdot \lambda_{l} k^{\sigma_{s} \sigma_{t}}, s t g\right) & & \left(\text { by }\left(C_{3}\right)\right) \\
& =\left(i j^{\sigma_{s}} \cdot l \cdot k^{\sigma_{s} \sigma_{t}}, s t g\right) & & \\
& =\left(i j^{\sigma_{s}} \cdot k^{\sigma_{s} \sigma_{t}}, s t g\right) & & \text { (since } \left.l, i j^{\sigma_{s}} \in I_{\alpha \beta}\right) \\
& =\left(i \cdot j^{\sigma_{s}} \cdot\left(k^{\sigma_{t}}\right)^{\sigma_{s}}, s t g\right) & & \\
& =\left(i\left(j k^{\sigma_{t}}\right)^{\sigma_{s}}, s(t g)\right) & & \text { (since } \left.\sigma_{s} \in \operatorname{End}(I)\right) \\
& =(i, s) \circ\left(j k^{\sigma_{t}}, t g\right) & & \\
& =(i, s) \circ[(j, t) \circ(k, g)] . &
\end{array}
$$

This shows that the multiplication "०" is associative.

By Lemma 4.1, we have already known that the set $S$ under the multiplication "०" is precisely a semigroup. Now, we give the following definition.

4.1. Definition. The semigroup constructed above is called a left twist product of a left regular band $I=\bigcup_{\alpha \in Y} I_{\alpha}$ and a $C$-a semigroup $T=\left[Y ; T_{\alpha} ; \theta_{\alpha \beta}\right]$ with respect to the semilattice $Y$, and is denoted by $I \ltimes_{Y} T$.

We now state the following main theorem of the paper.

4.2. Theorem. Let $Y$ be a semilattice. Let $T=\left[Y ; T_{\alpha} ; \theta_{\alpha, \beta}\right]$ be a strongly semilattice of cancellative monoids $T_{\alpha}(\alpha \in Y)$ and let $I=\bigcup_{\alpha \in Y} I_{\alpha}$ be a semilattice decomposition of a left regular band $I$ into left zero bands $I_{\alpha}$. Then, a left twist product $I \ltimes_{Y} T$ of $I$ and $T$ with respect to the semilattice $Y$ is a left regular cyber-group.

Conversely, every left regular cyber-group $S$ is isomorphic to one so constructed.

To prove the necessity part of Theorem 4.3, we need Definition 3.1 and also the following lemmas.

4.3. Lemma. Let $E$ be the set of all idempotents of the semigroup $I \ltimes_{Y} T$. Then $E=\left\{\left(i, e_{\alpha}\right) \mid i \in I_{\alpha}, e_{\alpha}\right.$ is the identity of $T_{\alpha}$ for each $\left.\alpha \in Y\right\}$ and $E$ is a left regular band.

Proof. We first check that every idempotent of the semigroup $S=I \ltimes_{Y} T$ can be expressed as the type $\left(i, e_{\alpha}\right)$ for some $\alpha \in Y$, where $e_{\alpha}$ is the identity of the cancellative $T_{\alpha}$. To see this, let $(i, s) \in S_{\alpha}$ for some $\alpha \in Y$. If $(i, s) \in E$, then by definition, $(i, s) \circ(i, s)=$ $\left(i i^{\sigma_{s}}, s^{2}\right)=(i, s)$. Consequently, $s^{2}=s$ in $T_{\alpha}$ and so the element $s$ must be the identity $e_{\alpha}$ of the cancelltive monoid $T_{\alpha}$. Hence, $(i, s)=\left(i, e_{\alpha}\right)$. Conversely, by the condition $\left(C_{1}\right)$, it follows that $\left(i, e_{\alpha}\right) \circ\left(i, e_{\alpha}\right)=\left(i i^{\sigma_{e_{\alpha}}}, e_{\alpha}^{2}\right)=\left(i, e_{\alpha}\right)$ and so $\left(i, e_{\alpha}\right) \in E$.

It remains to prove that $E$ is a left regular band. We consider a mapping $\varphi: E \longrightarrow I$ given by $\left(i, e_{\alpha}\right) \mapsto i$. It is evident that $\varphi$ is a bijection. In fact, the mapping $\varphi$ is also a homomorphism, for if $\left(i, e_{\alpha}\right),\left(j, e_{\beta}\right) \in E$, then we have the following equalities:

$$
\begin{aligned}
{\left[\left(i, e_{\alpha}\right) \circ\left(j, e_{\beta}\right)\right] \varphi } & =\left(i j^{\sigma_{e_{\alpha}}}, e_{\alpha} e_{\beta}\right) \varphi \\
& =i j^{\sigma_{e_{\alpha}}} \\
& =i 1_{\alpha} \cdot j \quad\left(1_{\alpha} \in I_{\alpha}, \text { by }\left(C_{2}\right)\right) \\
& =i j=\left(i, e_{\alpha}\right) \varphi\left(j, e_{\beta}\right) \varphi
\end{aligned}
$$


This shows that $\varphi$ is an isomorphism and so $E \simeq I$. By hypothesis, $E$ become a left regular band.

4.4. Lemma. The semigroup $S=I \ltimes_{Y} T$ constructed above is a superabundant semigroup.

Proof. To see that $S$ constructed above is superabundant, we let $a=(i, s) \in S_{\alpha}$ for some $\alpha \in Y$. We only need to prove that there exists an idempotent $a^{0} \in E$ such that $a \mathcal{H}^{*} a^{0}$. Putting $a^{0}=\left(i, e_{\alpha}\right)$, we will verify that $a \mathcal{L}^{*} a^{0}$ and $a \mathcal{R}^{*} a^{0}$. Assume that $u=(j, g) \in S_{\beta}^{1}$ and $v=(k, h) \in S_{\gamma}^{1}$. If $a \circ u=a \circ v$, then it can be easily verified that $\left(i j^{\sigma_{s}}, s g\right)=\left(i k^{\sigma_{s}}, s h\right)$, which implies that $i j^{\sigma_{s}}=i k^{\sigma_{s}}$ and $s g=s h$. By applying the condition $\left(C_{4}\right)$, it follows that $i j^{\sigma_{\alpha}}=i k^{\sigma_{e_{\alpha}}}$ and $e_{\alpha} g=e_{\alpha} h$. Hence, $a^{0} \circ u=$ $\left(i, e_{\alpha}\right) \circ(j, g)=\left(i j^{\sigma_{e_{\alpha}}}, e_{\alpha} g\right)=\left(i k^{\sigma_{e_{\alpha}}}, e_{\alpha} h\right)=\left(i, e_{\alpha}\right) \circ(k, h)=a^{0} \circ v$. Furthermore, we have $a \circ a^{0}=(i, s) \circ\left(i, e_{\alpha}\right)=\left(i i^{\sigma_{s}}, s e_{\alpha}\right)=(i, s)=a$. It follows from Corollary 2.2 that $a \mathcal{L}^{*} a^{0}$.

Similarly, notice the fact that $T=\left[Y ; T_{\alpha} ; \theta_{\alpha \beta}\right]$, we can easily check that $a \mathcal{R}^{*} a^{0}$ and so $a^{0}$ is precisely the identity of $H_{a}{ }^{*}$-class for every $a \in S$. This shows that $S$ is indeed a superabundant semigroup.

Using Definition 3.1 and summing up Lemma 4.1, Lemma 4.4 and Lemma 4.5, we have already proved the first part of Theorem 4.3 .

We now turn to prove the sufficiency part of Theorem 4.3. we first observe the following properties of a left regular cyber-group.

Suppose that $S$ is a left regular cyber-group. By virtue of Theorem 3.2, there exists a semilattice $Y$ such that $S=\left(Y ; S_{\alpha}\right)$, where each $S_{\alpha}=I_{\alpha} \times T_{\alpha}$ is a left rectangular monoid, that is, $S$ is a semilattice $Y$ of left rectangular monoids $S_{\alpha}=I_{\alpha} \times T_{\alpha}(\alpha \in Y)$. It is easy to see that

$$
E(S)=\left\{\left(i, e_{\alpha}\right) \mid i \in I_{\alpha}, e_{\alpha} \text { is the identity of } T_{\alpha} \text { for every } \alpha \in Y\right\} .
$$

Now, we choose a fixed element $u_{\alpha}=\left(1_{\alpha}, e_{\alpha}\right) \in E(S)$ for every $\alpha \in Y$. Furthermore, we let

$$
I=\bigcup_{\alpha \in Y} I_{\alpha} \quad \text { and } \quad T=\bigcup_{\alpha \in Y} T_{\alpha}
$$

We now prove the following lemmas.

4.5. Lemma. Let $S=\left(Y ; S_{\alpha}\right)$ be a left regular cyber-group. If a multiplication on the set $I=\bigcup_{\alpha \in Y} I_{\alpha}$ is defined as follows: for any $i \in I_{\alpha}, j \in I_{\beta}$,

$$
i j=k \quad \Leftrightarrow \quad\left(i, e_{\alpha}\right)\left(j, e_{\beta}\right)=\left(k, e_{\alpha \beta}\right),
$$

where $\left(i, e_{\alpha}\right),\left(j, e_{\beta}\right) \in E(S)$, then $I$ becomes a left regular band.

Proof. Consider the mapping $\phi: E(S) \longrightarrow I$ given by $\left(i, e_{\alpha}\right) \mapsto i$. It is easy to see that $\phi$ is a bijection. By Theorem 3.2, for any $\left(i, e_{\alpha}\right),\left(j, e_{\beta}\right) \in E(S)$, there exists some $k \in I_{\alpha \beta}$ such that $\left(i, e_{\alpha}\right)\left(j, e_{\beta}\right)=\left(k, e_{\alpha \beta}\right)$. Hence, By hypothesis, for any $i \in I_{\alpha}$ and $j \in I_{\beta}$, we define $i j=k$ if and only if $\left(i, e_{\alpha}\right)\left(j, e_{\beta}\right)=\left(k, e_{\alpha \beta}\right)$ for $\left(i, e_{\alpha}\right),\left(j, e_{\beta}\right) \in E(S)$. Consequently, $\left[\left(i, e_{\alpha}\right)\left(j, e_{\beta}\right)\right] \phi=\left(k, e_{\alpha \beta}\right) \phi=\left(i j, e_{\alpha \beta}\right) \phi=i j=\left(i, e_{\alpha}\right) \phi\left(j, e_{\beta}\right) \phi$. This shows that $\phi$ is an isomorphism and hence $I$ is a left regular band because $E(S)$ is a left regular band.

4.6. Lemma. Suppose that $S=\left(Y ; S_{\alpha}\right)$ is a left regular cyber-group and $T=\bigcup_{\alpha \in Y} T_{\alpha}$. Let $\theta_{\alpha, \beta}$ be the mapping from $T_{\alpha}$ to $T_{\beta}$ defined by the rule that for any $\alpha, \beta \in Y$ with $\alpha \geqslant \beta$ :

$$
\theta_{\alpha, \beta}: s \mapsto s \theta_{\alpha, \beta} \quad \Leftrightarrow \quad\left(1_{\beta}, e_{\beta}\right)\left(1_{\alpha}, s\right)=\left(1_{\beta}, s \theta_{\alpha, \beta}\right)
$$


where $\left(1_{\beta}, e_{\beta}\right) \in S_{\beta}$ and $\left(1_{\alpha}, s\right) \in S_{\alpha}$. Then $T=T\left(Y ; T_{\alpha} ; \theta_{\alpha, \beta}\right)$ becomes a strong semilattice of $T_{\alpha}$ with homomorphisms $\theta_{\alpha, \beta}$. Furthermore, for any $(i, s) \in S_{\alpha},(j, t) \in S_{\beta}$, we have the following equality:

$$
(i, s)(j, t)=(k, s t)
$$

for some $k \in I_{\alpha \beta}$.

Proof. Suppose that $S=\left(Y ; S_{\alpha}\right)$ is a left regular cyber-group, $\left(1_{\beta}, e_{\beta}\right) \in S_{\beta},\left(1_{\alpha}, s\right) \in S_{\alpha}$. Let $T=\bigcup_{\alpha \in Y} T_{\alpha}, 1_{\beta} \in I_{\beta}$, and $1_{\alpha} \in I_{\alpha}$ such that $1_{\alpha} 1_{\beta}=1_{\beta}$ for $\alpha \geqslant \beta$, by Lemma 4.6. Now define a mapping from $T_{\alpha}$ to $T_{\beta}$ by the rule that for $\alpha \geqslant \beta$,

$$
\theta_{\alpha, \beta}: s \mapsto s \theta_{\alpha, \beta} \Leftrightarrow\left(1_{\beta}, e_{\beta}\right)\left(1_{\alpha}, s\right)=\left(1_{\beta}, s \theta_{\alpha, \beta}\right) .
$$

It is easy to see that $\theta_{\alpha, \alpha}$ is the identical automorphism of $T_{\alpha}$ for each $\alpha \in Y$. Let $\alpha, \beta \in Y$ be such that $\alpha \geqslant \beta$ and let $\left(1_{\alpha}, s\right),\left(1_{\alpha}, t\right) \in S_{\alpha}$. Then, we have

$$
\begin{aligned}
\left(1_{\beta}, e_{\beta}\right)\left[\left(1_{\alpha}, s\right)\left(1_{\alpha}, t\right)\right] & =\left(1_{\beta}, e_{\beta}\right)\left(1_{\alpha}, s t\right) \\
& =\left(1_{\beta},(s t) \theta_{\alpha, \beta}\right)
\end{aligned}
$$

and

$$
\begin{aligned}
{\left[\left(1_{\beta}, e_{\beta}\right)\left(1_{\alpha}, s\right)\right]\left(1_{\alpha}, t\right) } & =\left(1_{\beta}, s \theta_{\alpha, \beta}\right)\left(1_{\alpha}, t\right) \\
& =\left(1_{\beta}, s \theta_{\alpha, \beta}\right)\left(1_{\beta}, e_{\beta}\right)\left(1_{\alpha}, t\right) \\
& =\left(1_{\beta}, s \theta_{\alpha, \beta}\right)\left[\left(1_{\beta}, e_{\beta}\right)\left(1_{\alpha}, t\right)\right] \\
& =\left(1_{\beta}, s \theta_{\alpha, \beta}\right)\left(1_{\beta}, t \theta_{\alpha, \beta}\right) \\
& =\left(1_{\beta}, s \theta_{\alpha, \beta} t \theta_{\alpha, \beta}\right) .
\end{aligned}
$$

Hence, $(s t) \theta_{\alpha, \beta}=s \theta_{\alpha, \beta} t \theta_{\alpha, \beta}$ and so $\theta_{\alpha, \beta}$ is a homomorphism from $T_{\alpha}$ to $T_{\beta}$.

Let $\alpha, \beta, \gamma \in Y$ be such that $\alpha \geqslant \beta \geqslant \gamma$ and let $\left(1_{\alpha}, s\right) \in S_{\alpha}$. Then,

$$
\begin{aligned}
\left(1_{\gamma}, s \theta_{\alpha, \gamma}\right) & =\left(1_{\gamma}, e_{\gamma}\right)\left(1_{\alpha}, s\right) \\
& =\left(1_{\gamma}, e_{\gamma}\right)\left(1_{\beta}, e_{\beta}\right)\left(1_{\alpha}, s\right) \quad \text { by Lemma 4.6) } \\
& =\left(1_{\gamma}, e_{\gamma}\right)\left(1_{\beta}, s \theta_{\alpha, \beta}\right) \\
& =\left(1_{\gamma},\left(s \theta_{\alpha, \beta}\right) \theta_{\beta, \gamma}\right) .
\end{aligned}
$$

Consequently, the mapping $\theta_{\alpha, \beta}$ defined by the formula (4.2) satisfies the properties $\theta_{\alpha, \gamma}=\theta_{\alpha, \beta} \theta_{\beta, \gamma}$. We have proved that $T=T\left(Y ; T_{\alpha} ; \theta_{\alpha, \beta}\right)$ is a strong semilattice of cancellative monoids $T_{\alpha}$ under the multiplication given by $s t=s \theta_{\alpha, \alpha \beta} t \theta_{\beta, \alpha \beta}$ for any $s \in T_{\alpha}, t \in T_{\beta}$.

To complete the remaining part of the proof, suppose that for any $(i, s) \in S_{\alpha}$ and $(j, t) \in S_{\beta}$,

$$
(i, s)(j, t)=(k, u)
$$

for some $k \in I_{\alpha \beta}, u \in T_{\alpha \beta}$. Then,

$$
\begin{aligned}
(k, u) & =\left(k, e_{\alpha \beta}\right)(k, u)\left(k, e_{\alpha \beta}\right) \\
& =\left(k, e_{\alpha \beta}\right)(i, s)(j, t)\left(k, e_{\alpha \beta}\right) \\
& =\left(k, e_{\alpha \beta}\right)\left(1_{\alpha \beta}, e_{\alpha \beta}\right)(i, s)(j, t)\left(k, e_{\alpha \beta}\right) \\
& =\left[\left(k, e_{\alpha \beta}\right)\left(1_{\alpha \beta}, e_{\alpha \beta}\right)(i, s)\right]\left(1_{\alpha \beta}, e_{\alpha \beta}\right)\left[(j, t)\left(k, e_{\alpha \beta}\right)\right] \quad \text { (by Lemma 3.3) } \\
& =\left(k, e_{\alpha \beta}\right)\left[\left(1_{\alpha \beta}, e_{\alpha \beta}\right)\left(1_{\alpha}, e_{\alpha}\right)\right](i, s)\left[\left(1_{\alpha \beta}, e_{\alpha \beta}\right)\left(1_{\beta}, e_{\beta}\right)\right](j, t)\left(k, e_{\alpha \beta}\right) \\
& \\
& =\left(k, e_{\alpha \beta}\right)\left(1_{\alpha \beta}, e_{\alpha \beta}\right)\left[\left(1_{\alpha}, e_{\alpha}\right)(i, s)\right]\left(1_{\alpha \beta}, e_{\alpha \beta}\right)\left[\left(1_{\beta}, e_{\beta}\right)(j, t)\right]\left(k, e_{\alpha \beta}\right) \\
& =\left(k, e_{\alpha \beta}\right)\left[\left(1_{\alpha \beta}, e_{\alpha \beta}\right)\left(1_{\alpha}, s\right)\right]\left[\left(1_{\alpha \beta}, e_{\alpha \beta}\right)\left(1_{\beta}, t\right)\right]\left(k, e_{\alpha \beta}\right) \\
& =\left(k, e_{\alpha \beta}\right)\left(1_{\alpha \beta}, s \theta_{\alpha, \alpha \beta}\right)\left(1_{\alpha \beta}, t \theta_{\beta, \alpha \beta}\right)\left(k, e_{\alpha \beta}\right) \\
& =\left(k, s \theta_{\alpha, \alpha \beta} t \theta_{\beta, \alpha \beta}\right) \\
& =(k, s t) .
\end{aligned}
$$


The proof is completed.

For any $s \in T_{\alpha}$ and $\alpha \in Y$ we define a mapping $\sigma_{s}: I \longrightarrow I$ given by the rule that for any $j \in I_{\beta}$ and $\beta \in Y$,

$$
\left(1_{\alpha}, s\right)\left(j, e_{\beta}\right)=\left(\sigma_{s}(j),-\right)
$$

where $e_{\beta}$ is the identity of a cancellative monoid $T_{\beta}$.

4.7. Lemma. Let $S=\left(Y ; S_{\alpha}\right)$ be a left regular cyber-group and let $\sigma_{s}$ be the mapping as given above by the formula (4.3). Then for any $i, j \in I, \sigma_{s}(i j)=\sigma_{s}(i) \sigma_{s}(j)$, that is, $\sigma_{s} \in$ End $(I)$.

Proof. Suppose that $S=\left(Y ; S_{\alpha}\right)$ be a left regular cyber-group. By Lemma 4.6 and Lemma 4.7, $I=\bigcup_{\alpha \in Y} I_{\alpha}$ and $T=\bigcup_{\alpha \in Y} T_{\alpha}$ forms a left regular band and a strong semilattice of cancellative monoids $T_{\alpha}$, respectively. For any $s \in T_{\gamma}$, let $\sigma_{s}$ be the mapping as given by the formula (4.3). Then for any $i \in I_{\alpha}$ and $j \in I_{\beta}$ it follows by Lemma 4.6 that $\left(1_{\gamma}, e_{\gamma}\right)\left(i, e_{\alpha}\right)\left(j, e_{\beta}\right)=\left(1_{\gamma} i j, e_{\alpha \beta \gamma}\right)$. Moreover, since $T$ is a $C$-a semigroup, it is clear from Lemma 2.4 that $s e_{\alpha}=e_{\alpha} s$ for any $s \in T$ and $e_{\alpha} \in E(T)$. Hence, by the formula (4.3), we deduce the following equities:

$$
\begin{array}{rlr}
\left(\sigma_{s}(i j), s e_{\alpha \beta}\right) & =\left(1_{\gamma}, s\right)\left(i j, e_{\alpha \beta}\right) & \\
& =\left(1_{\gamma}, s\right)\left(i, e_{\alpha}\right)\left(j, e_{\beta}\right) & \\
& =\left(1_{\gamma}, s\right)\left(1_{\gamma}, e_{\gamma}\right)\left(i, e_{\alpha}\right)\left(j, e_{\beta}\right) & \\
& =\left(1_{\gamma}, s\right)\left[\left(1_{\gamma}, e_{\gamma}\right)\left(i, e_{\alpha}\right)\left(j, e_{\beta}\right)\right]^{2} & \\
& =\left(1_{\gamma}, s\right)\left(i, e_{\alpha}\right)\left(j, e_{\beta}\right)\left(1_{\gamma} i j, e_{\alpha \beta \gamma}\right) \quad(\text { by Lemma 4.6) } \\
& =\left[\left(1_{\gamma}, s\right)\left(i, e_{\alpha}\right)\right]\left(j, e_{\beta}\right)\left(1_{\gamma} i j, e_{\alpha \beta \gamma}\right) & \\
& =\left(\sigma_{s}(i), s e_{\alpha}\right)\left(j, e_{\beta}\right)\left(1_{\gamma} i j, e_{\alpha \beta \gamma}\right) \quad(\text { by }(4.2) & \text { and }(4.3)) \\
& =\left(\sigma_{s}(i), e_{\alpha \gamma}\right)\left(1_{\alpha \gamma}, s e_{\alpha}\right)\left(j, e_{\beta}\right)\left(1_{\gamma} i j, e_{\alpha \beta \gamma}\right) \\
& =\left(\sigma_{s}(i), e_{\alpha \gamma}\right)\left(1_{\alpha \gamma}, e_{\alpha} s\right)\left(j, e_{\beta}\right)\left(1_{\gamma} i j, e_{\alpha \beta \gamma}\right) \\
& =\left(\sigma_{s}(i), e_{\alpha \gamma}\right)\left(1_{\alpha}, e_{\alpha}\right)\left(1_{\gamma}, s\right)\left(j, e_{\beta}\right)\left(1_{\gamma} i j, e_{\alpha \beta \gamma}\right) \\
& =\left(\sigma_{s}(i), e_{\alpha \gamma}\right)\left[\left(1_{\alpha}, e_{\alpha}\right)\left(1_{\gamma}, e_{\gamma}\right)\right]\left(1_{\gamma}, s\right)\left(j, e_{\beta}\right)\left(1_{\gamma} i j, e_{\alpha \beta \gamma}\right) \\
& =\left(\sigma_{s}(i), e_{\alpha \gamma}\right)\left(1_{\alpha \gamma}, e_{\alpha \gamma}\right)\left[\left(1_{\gamma}, s\right)\left(j, e_{\beta}\right)\right]\left(1_{\gamma} i j, e_{\alpha \beta \gamma}\right) \\
& =\left(\sigma_{s}(i), e_{\alpha \gamma}\right)\left(\sigma_{s}(j), s e_{\beta}\right)\left(1_{\gamma} i j, e_{\alpha \beta \gamma}\right) \\
& =\left[\left(\sigma_{s}(i), e_{\alpha \gamma}\right)\left(\sigma_{s}(j), e_{\gamma \beta}\right)\right]\left[\left(1_{\gamma \beta}, s e_{\beta}\right)\left(1_{\gamma} i j, e_{\alpha \beta \gamma}\right)\right] \\
& =\left(\sigma_{s}(i) \sigma_{s}(j), e_{\alpha \beta \gamma}\right)\left(-, s e_{\alpha \beta}\right) \quad(\text { by }(4.2) \text { and Lemma 4.6) } \\
& =\left(\sigma_{s}(i) \sigma_{s}(j), s e_{\alpha \beta}\right) .
\end{array}
$$

Hence, $\sigma_{s}(i j)=\sigma_{s}(i) \sigma_{s}(j)$ and so $\sigma_{s} \in$ End $(I)$, as required.

4.8. Lemma. Suppose that $S=\left(Y ; S_{\alpha}\right)$ is a left regular cyber-group. Let $I$ and $T=$ $T\left(Y ; T_{\alpha} ; \varphi_{\alpha, \beta}\right)$ be a left regular band and a strong semilattice of cancellative monoids $T_{\alpha}$, respectively. Then the mapping $\sigma: T \longrightarrow$ End $(I)$ defined by $s \mapsto \sigma_{s}$ satisfies the previous conditions $\left(C_{1}\right)-\left(C_{4}\right)$, where $\sigma_{s}$ is given by the formula (4.3).

Proof. By the formulae (4.3) and (4.2), it follows directly that

$$
\left(1_{\alpha}, s\right)\left(j, e_{\beta}\right)=\left(\sigma_{s}(j), s e_{\beta}\right)
$$

for any $s \in T_{\alpha}, j \in I_{\beta}$, and any $\alpha, \beta \in Y$. From the formula (4.4), it is easy to see that the mapping $\sigma: T \rightarrow \operatorname{End}(I)$ given by $s \mapsto \sigma_{s}$ satisfies the condition $\left(C_{1}\right)$ and $\left(C_{2}\right)$. To see that the condition $\left(C_{3}\right)$ is satisfied by the mapping $\sigma$, suppose that $s \in T_{\alpha}, t \in T_{\beta}, i \in I_{\alpha \beta}$ and $j \in I_{\gamma}$. Then, by Lemma 4.6 and Lemma 4.7 we have 


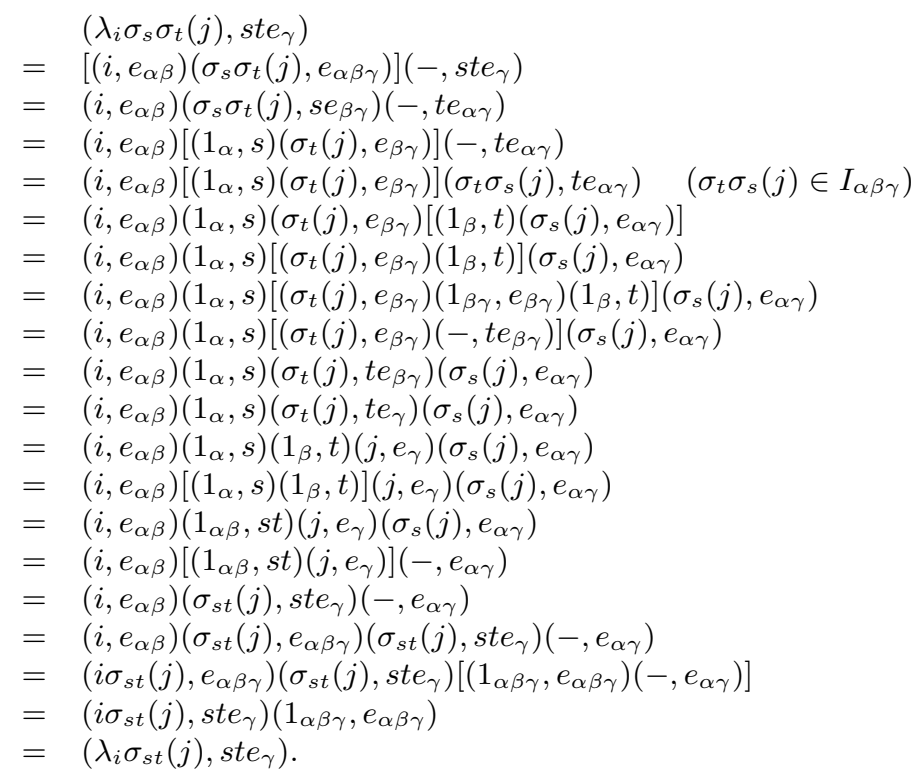

This shows that $\lambda_{i} \sigma_{s} \sigma_{t}(j)=\lambda_{i} \sigma_{s t}(j)$ and so the condition $\left(C_{3}\right)$ is satisfied by the mapping $\sigma$.

To show that the mapping $\sigma$ satisfies the condition $\left(C_{4}\right)$, we notice the fact that for any $u=(i, a) \in S_{\alpha}=I_{\alpha} \times T_{\alpha}$, there exists a unique element $u^{0}=\left(i, e_{\alpha}\right) \in E(S) \cap S_{\alpha}$ such that $u \mathcal{L}^{*} u^{0}$ and $u \mathcal{R}^{*} u^{0}$ in the left regular cyber-group $S$. Suppose that $(j, s) \in S_{\beta}=$ $I_{\beta} \times T_{\beta},(k, t) \in S_{\gamma}=I_{\gamma} \times T_{\gamma}$ such that $(i, a)(j, s)=(i, a)(k, t)$, that is, $i \sigma_{a}(j)=i \sigma_{a}(k)$ and $a s=a t$. Then, since $u \mathcal{L}^{*} u^{0}$, it follows by Lemma 2.1 that $\left(i, e_{\alpha}\right)(j, s)=\left(i, e_{\alpha}\right)(k, t)$ which implies $i \sigma_{e_{\alpha}}(j)=i \sigma_{e_{\alpha}}(k)$ and $e_{\alpha} s=e_{\alpha} t$. Consequently, the condition $\left(C_{4}\right)$ is satisfied. This complete the proof.

The Proof of Theorem 4.3 Finally, we return to the proof of Theorem 4.3. Suppose that $S=\left(Y ; S_{\alpha}\right)$ is a left regular cyber-group. Then, by Lemma 4.6, Lemma 4.7, Lemma 4.8, and Lemma 4.9, we have already known that there exist two semigroups $I$ and $T$, where $I$ is a left regular band and $T$ is a strong semilattice of cancellative monoids, and also there exists a mapping $\sigma$ from $T$ to End $(I)$ given by the formula (4.3) satisfying the conditions $\left(C_{1}\right)-\left(C_{4}\right)$. Thus, by our definition, we directly obtain the left twist product $I \ltimes_{Y} T$ of $I$ and $T$ with respect to semilattice $Y$. To complete the proof of the second part of Theorem 4.3, we only need to show that the multiplication of $S$ coincides with that of the left twist product $I \ltimes_{Y} T$ so that $S \simeq I \ltimes_{Y} T$. Hence, we let $(i, s) \in S_{\alpha}$ and $(j, t) \in S_{\beta}$. Then, using Lemma 4.6, Lemma 4.7, and the formula (4.4) we deduce that

$$
\begin{aligned}
(i, s)(j, t) & =\left(i, e_{\alpha}\right)\left(1_{\alpha}, s\right)\left(j, e_{\beta}\right)\left(1_{\beta}, t\right) \\
& =\left(i, e_{\alpha}\right)\left[\left(1_{\alpha}, s\right)\left(j, e_{\beta}\right)\right]\left(1_{\beta}, t\right) \\
& =\left(i, e_{\alpha}\right)\left(\sigma_{s}(j), s e_{\beta}\right)\left(1_{\beta}, t\right) \\
& =\left(i, e_{\alpha}\right)\left(\sigma_{s}(j), e_{\alpha \beta}\right)\left(1_{\alpha \beta}, s e_{\beta}\right)\left(1_{\beta}, t\right) \\
& =\left(i \sigma_{s}(j), e_{\alpha \beta}\right)(-, s t) \\
& =\left(i \sigma_{s}(j), s t\right) \\
& =\left(i j^{\sigma_{s}}, s t\right)
\end{aligned}
$$

where $\sigma_{s}(j)=j^{\sigma_{s}}$. Consequently, the proof is now completed.

Remark: In the construction of a left regular cyber-group $S=I \ltimes_{Y} T$, if we let $T=\left[Y ; T_{\alpha} ; \theta_{\alpha, \beta}\right]$ be a strongly semilattice of groups $T_{\alpha}(\alpha \in Y)$, that is, $T$ is a Clifford 
semigroup, then we can modify our requirement condition $\left(C_{1}\right)-\left(C_{4}\right)$ to $\left(C_{1}\right)-\left(C_{3}\right)$. In this case, the semigroup $S=I \ltimes_{Y} T$ will become a completely regular semigroup in which its idempotents is a left regular band, that is, a left regular orthogroup (detail,see [9]). Thus the known structure theorem given by M.Yamada and M.Petrich of left regular orthogroups now follows as a direct consequence of our Theorem 4.3.

\section{A constructed left regular cyber-group}

In this section, we construct an example of a non-trivial left regular cyber-groups by using the method given in the above section.

Let $Y=\{\alpha, \beta\}$ be a semilattice such that $\alpha \geqslant \beta$. Let $I=I_{\alpha} \cup I_{\beta}$ be a left regular band with the following Cayley table, where $I_{\alpha}=\left\{e_{1}, e_{2}\right\}$ and $I_{\beta}=\left\{e_{3}, e_{4}\right\}$ are respectively left zero bands.

\begin{tabular}{l|llll}
$*$ & $e_{1}$ & $e_{2}$ & $e_{3}$ & $e_{4}$ \\
\hline$e_{1}$ & $e_{1}$ & $e_{1}$ & $e_{4}$ & $e_{4}$ \\
$e_{2}$ & $e_{2}$ & $e_{2}$ & $e_{4}$ & $e_{4}$ \\
$e_{3}$ & $e_{3}$ & $e_{3}$ & $e_{3}$ & $e_{3}$ \\
$e_{4}$ & $e_{4}$ & $e_{4}$ & $e_{4}$ & $e_{4}$
\end{tabular}

Furthermore, assume that $T_{\alpha}=\left\{s_{0}, s_{1}, s_{2}\right\}$ is a group with the identity element $s_{0}$ and $A$ is the matrix $\left(\begin{array}{ll}1 & 0 \\ 1 & 0\end{array}\right)$. Then $T_{\beta}=\left\{t_{n}=2^{n} A \mid n \geqslant 0 \& n \in N\right\}$ under the matrix multiplication forms an infinite cancellative monoid. Let $T=T_{\alpha} \cup T_{\beta}$ and define a multiplication on $T$ which extends on $T_{\alpha}$ and $T_{\beta}$ as follows : $x y=y x=y$ for all $x \in T_{\alpha}, y \in T_{\beta}$. It is routine to check that $T$ is a strong semilattice of cancellative monoids $T_{\alpha}(\alpha \in Y)$, that is, $T=T\left[Y ; T_{\alpha} ; \theta_{\alpha, \beta}\right]$, a $C$ - $a$ semigroup.

By using the above constructed $I$ and $T$, we form the set $S=S_{\alpha} \cup S_{\beta}=\left\{a, b, c, d, e, f, u_{0}, v_{0}, u_{n}, v_{m} \mid n, m \in N\right\}$, where the elements have the following expressions:

$$
\begin{aligned}
& a=\left(e_{1}, s_{0}\right), \quad b=\left(e_{2}, s_{0}\right), \quad c=\left(e_{1}, s_{1}\right), \quad d=\left(e_{2}, s_{1}\right), \quad e=\left(e_{1}, s_{2}\right) \\
& f=\left(e_{2}, s_{2}\right), \quad u_{0}=\left(e_{3}, t_{0}\right), \quad v_{0}=\left(e_{4}, t_{0}\right), \quad u_{n}=\left(e_{3}, t_{n}\right), \quad v_{n}=\left(e_{4}, t_{n}\right)
\end{aligned}
$$

for $n=1,2, \cdots$.

As described in Section 3, to obtain the left twist product $I \ltimes_{Y} T$ of the left regular band $I$ and the $C$ - $a$ semigroup $T$, we need to define a mapping

$$
\sigma: T \longrightarrow \text { End }(I)
$$

by $s \mapsto \sigma_{s}$ as follows:

$$
\begin{aligned}
& \sigma_{s_{0}}=\left(\begin{array}{cccc}
e_{1} & e_{2} & e_{3} & e_{4} \\
e_{1} & e_{1} & e_{4} & e_{4}
\end{array}\right), \quad \sigma_{s_{1}}=\left(\begin{array}{cccc}
e_{1} & e_{2} & e_{3} & e_{4} \\
e_{1} & e_{1} & e_{3} & e_{4}
\end{array}\right) \\
& \sigma_{s_{2}}=\left(\begin{array}{cccc}
e_{1} & e_{2} & e_{3} & e_{4} \\
e_{2} & e_{2} & e_{4} & e_{4}
\end{array}\right), \quad \sigma_{t_{0}}=\left(\begin{array}{llll}
e_{1} & e_{2} & e_{3} & e_{4} \\
e_{3} & e_{3} & e_{3} & e_{3}
\end{array}\right), \\
& \sigma_{t_{n}}=\left(\begin{array}{cccc}
e_{1} & e_{2} & e_{3} & e_{4} \\
e_{4} & e_{4} & e_{4} & e_{4}
\end{array}\right)
\end{aligned}
$$

where $n=1,2, \cdots$.

It is easy to check that the mapping $\sigma: T \longrightarrow$ End $(I)$ satisfies the conditions $\left(C_{1}\right)$ $\left(C_{4}\right)$ in the left twist product of semigroups $I$ and $T$. By using the left twist product of $I$ and $T$, as described above, we put the product of the semigroup $I \ltimes_{Y} T$ as follows: for any $(i, s),(j, t) \in S$,

$$
(i, s) \circ(j, t)=\left(i j^{\sigma_{s}}, s t\right)
$$


where $j^{\sigma_{s}}=\sigma_{s}(j)$. Consequently, by using the above defined multiplication, we obtain the following Cayley table for the semigroup $S=I \ltimes_{Y} T$, the left twist produce of semigroups $I$ and $T$.

\begin{tabular}{l|llllllllll}
$*$ & $a$ & $b$ & $c$ & $d$ & $e$ & $f$ & $u_{0}$ & $v_{0}$ & $u_{l}$ & $v_{m}$ \\
\hline$a$ & $a$ & $a$ & $c$ & $c$ & $e$ & $e$ & $v_{0}$ & $v_{0}$ & $v_{l}$ & $v_{m}$ \\
$b$ & $b$ & $b$ & $d$ & $d$ & $f$ & $f$ & $v_{0}$ & $v_{0}$ & $v_{l}$ & $v_{m}$ \\
$c$ & $c$ & $c$ & $e$ & $e$ & $a$ & $a$ & $v_{0}$ & $v_{0}$ & $v_{l}$ & $v_{m}$ \\
$d$ & $d$ & $d$ & $f$ & $f$ & $b$ & $b$ & $v_{0}$ & $v_{0}$ & $v_{l}$ & $v_{m}$ \\
$e$ & $e$ & $e$ & $a$ & $a$ & $c$ & $c$ & $v_{0}$ & $v_{0}$ & $v_{l}$ & $v_{m}$ \\
$f$ & $f$ & $f$ & $b$ & $b$ & $d$ & $d$ & $v_{0}$ & $v_{0}$ & $v_{l}$ & $v_{m}$ \\
$u_{0}$ & $u_{0}$ & $u_{0}$ & $u_{0}$ & $u_{0}$ & $u_{0}$ & $u_{0}$ & $u_{0}$ & $u_{0}$ & $u_{l}$ & $u_{m}$ \\
$v_{0}$ & $v_{0}$ & $v_{0}$ & $v_{0}$ & $v_{0}$ & $v_{0}$ & $v_{0}$ & $v_{0}$ & $v_{0}$ & $v_{l}$ & $v_{m}$ \\
$u_{k}$ & $u_{k}$ & $u_{k}$ & $u_{k}$ & $u_{k}$ & $u_{k}$ & $u_{k}$ & $u_{k}$ & $u_{k}$ & $u_{k+l}$ & $u_{k+m}$ \\
$v_{n}$ & $v_{n}$ & $v_{n}$ & $v_{n}$ & $v_{n}$ & $v_{n}$ & $v_{n}$ & $v_{n}$ & $v_{n}$ & $v_{n+l}$ & $v_{n+m}$
\end{tabular}

where $k, l, m, n=1,2,3, \cdots$.

By Theorem 4.3, it can be verified that the above constructed semigroup $S=I \ltimes_{Y} T$ is an infinite left regular cyber-group. It is worth to note that, in our verification that $S=I \ltimes_{Y} T$ is a semigroup, there is no need to check that the associative law holds in the Cayley table. The associativity follows from the definition of left twist produce of semigroups.

In the above Cayley table, we can see that $E(S)=\left\{a, b, u_{0}, v_{0}\right\}$ is the set of all idempotents of the semigroup $S$. By Lemma 2.1 and its dual for $\mathcal{R}^{*}$, we can easily verify that $\mathcal{L}^{*}$-classes of $S$ are the sets $\{a, b, c, d, e, f\}$ and $\left\{u_{0}, v_{0}, u_{n}, v_{n} \mid n \in N\right\}$. The $\mathcal{R}^{*}$ classes of $S$ are the sets $\{a, c, e\} ;\{b, d, f\}$;

$\left\{u_{0}, u_{n} \mid n \in N\right\}$ and $\left\{v_{0}, v_{n} \mid n \in N\right\}$ respectively. Hence, the $\mathcal{H}^{*}$-classes of $S$ are the sets $\{a, c, e\} ;\{b, d, f\} ;\left\{u_{0}, u_{n} \mid n \in N\right\}$ and $\left\{v_{0}, v_{n} \mid n \in N\right\}$. Clearly, each $\mathcal{H}^{*}$-class of $S$ contains an idempotent and so $S$ is a superabundant semigroup. In fact, by definition $S$ is precisely a left regular cyber-group because the set of all idempotents of $S$ forms a left regular band.

It is easy to see that $S_{1}=\left\{a, b, c, d, e, f, u_{0}, v_{0}\right\}$ is a subsemigroup of $S$, which is completely regular and in which the set of all idempotents forms a left regular band. Also we know that every element of $S \backslash S_{1}$ is non-regular. This shows that $S_{1}$ is a left regular orthogroup (see [9]) and also the class of left regular orthogroups are a proper subclasses of the class of left regular cyber-groups. More complicated left regular cybergroups can also be constructed by using this method.

\section{References}

[1] G. L. Bailes, Right inverse semigroups, J. of Algebra 26 (1973), 492-507.

[2] A. El-Qallali and J. B. Fountain, Quasi-adequate semigroups, Proc. Roy. Soc. Edinburgh Sect. A, (1981), 91-99.

[3] J. B. Fountain, Adequate semigroups, Proc. Edinburgh Math. Soc. 22 (1979), 113-125.

[4] J. B. Fountain, Abundant semigroups, Proc. Lond. Math. Soc. 44 (1982), 103-129.

[5] X. J. Guo and K.P.Shum, On left cyber groups, Intern. Math. Journal 5(8) (2004), 705-717.

[6] J. M. Howie, An introduction to semigroup theory, Academic Press, London, 1976.

[7] J. M. Howie, Fundamentals of semigroup theory, Oxford University Press, New York, 1995.

[8] X.Z. Kong and K.P. Shum, Semilattice structure of rgular cyber groups, Pragmetic Algebra I, India, (2006), 1-12.

[9] M. Petrich and N. R. Reilly, Completely regular semigroups, John Wiley \& Sons, New York, 1999. 
[10] M. Petrich, A structure theorem for completely regular semigroups, Proc. Amer. Math. Soc., 99 (4) (1987), 617-622.

[11] X. M. Ren and K. P. Shum, The structure of $Q^{*}$-inverse semigroups, J. of Algebra, 325 (2011), 1-17.

[12] X. M. Ren and K. P. Shum, The structure of superabundant semigroups, Science in China Series A: Mathematics 47(5) (2004), 756-771.

[13] X. M. Ren and K. P. Shum, On superabundant semigroups whose set of idempotents forms a subsemigroup, Algebra Colloquium 14:2 (2007), 215-228.

[14] X. M. Ren and K. P. Shum, The structure of $\mathcal{L}^{*}$-inverse semigroups, Science in China Series A: Mathematics 49(8) (2006), 1065-1081.

[15] K. P. Shum, X. M. Ren and Y. Q. Guo, On $\mathcal{C}^{*}$-quasiregular semigroups, Communications in Algebra, 27 (19) (1999), 4251-4274.

[16] Lili Wang and Aifa Wang, Some properties of regular crypto H-abundant groups, Proceedings of The 3rd International conference in Electric and Electronic, (EEJC-13), Atlantis Press, doi:10.2991/eeic-13-2013-97.

[17] P. S. Venkatsen, Right (left) inverse semigroups, J. Algebra 31 (1974), 209-217.

[18] M. Yamada, Orthodox semigroups whose idempotents satisfy a certain identity, Semigroup Forum 6 (1973), 113-128. 